Supporting Information:

\title{
Mechanism by which aluminium regulates the abnormal phosphorylation of the Tau protein in different cell lines
}

Baolong Pan ${ }^{1,2^{*}}$, Xiaoting $\mathrm{Lu}^{1 *}$, Xiao Han ${ }^{1}$, Jiaping Huan ${ }^{1}$, Dan Gao ${ }^{1}$, Shuangjie Cui ${ }^{1}$, Xiaofen $\mathrm{Ju}^{1}$, Yunwei Zhang ${ }^{1}$, Shimeng $\mathrm{Xu}^{1}$, Jing Song ${ }^{1}$, Linping Wang ${ }^{1}$, Huifang Zhang ${ }^{1}$, Qiao Niu ${ }^{1}$

1- Department of Occupational Health, School of Public Health, Shanxi Medical University, China

2- Sixth Hospital of Shanxi Medical University (General hospital of Tisco), China

\#First Authors: Pan Baolong, Lu Xiaoting

*Corresponding author: Lu Xiaoting, luxiaoting@sxmu.edu.cn, Pan Baolong, 2057749336@qq.com.

Table S1 Characteristics of the primary antibodies used in the experiment.

.. $\mathrm{S} 2$

Figure S1 The expression of tau-5 and phosphorylated Tau protein induced by $\mathrm{AlCl} 3$ in $\mathrm{N} 2 \mathrm{a}$ cells.

Figure S2 The expression of tau-5 and phosphorylated Tau in SH-SY5Y

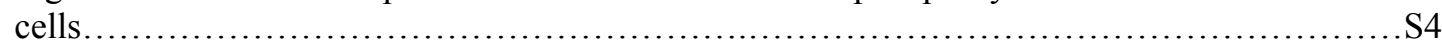

Figure S3 The levels of GSK-3 $\beta$ and PP2A induced by AlCl3 in N2a \& SH-SY5Y cells.

Figure S4 The expression of CHIP, HSP70, and the level of Ub in aluminium-exposed N2A cells. .S6

Figure S5 The expression of CHIP, HSP70, and the levels of Ub in aluminium-exposed SH-SY5Y cells.

Figure S6 The expression of total and phosphorylated Tau in N2a cells treated with MG132. S10

Figure S7 The expression of total and phosphorylated Tau in SH-SY5Y cells treated with MG132.

Figure S8 The expression of CHIP and Hsp70, and the level of Ub, in UPP of N2a cells treated with MG132..... 
Figure S9 The expression of CHIP and Hsp70, and the levels of Ub, in the UPP of SH-SY5Y cells

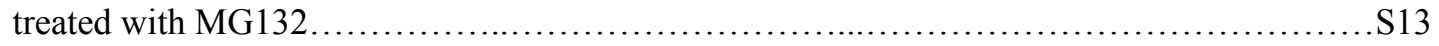

Table S1 Characteristics of the primary antibodies used in the experiment

\begin{tabular}{cccc}
\hline Primary antibodies & Parasitifer & Manufacturer & Dilution Rate \\
\hline tau-5 & mice & Abcam & $1: 1000$ \\
pThr181 & rabbit & Gene Tex & $1: 1000$ \\
pThr231 & rabbit & Abcam & $1: 1500$ \\
pSer262 & rabbit & Gene Tex & $1: 1000$ \\
pSer396 & rabbit & Abcam & $1: 5000$ \\
CHIP & rabbit & Abcam & $1: 2000$ \\
HSP70 & mice & Gene Tex & $1: 1000$ \\
Ub & rabbit & Abcam & $1: 1000$ \\
GADPH & rabbit & Beijing Kangwei & $1: 3000$ \\
& & Century Company & $1: 3000$ \\
$\beta$-actin & mice & Beijing Kangwei & \\
& & Century Company & \\
\hline
\end{tabular}




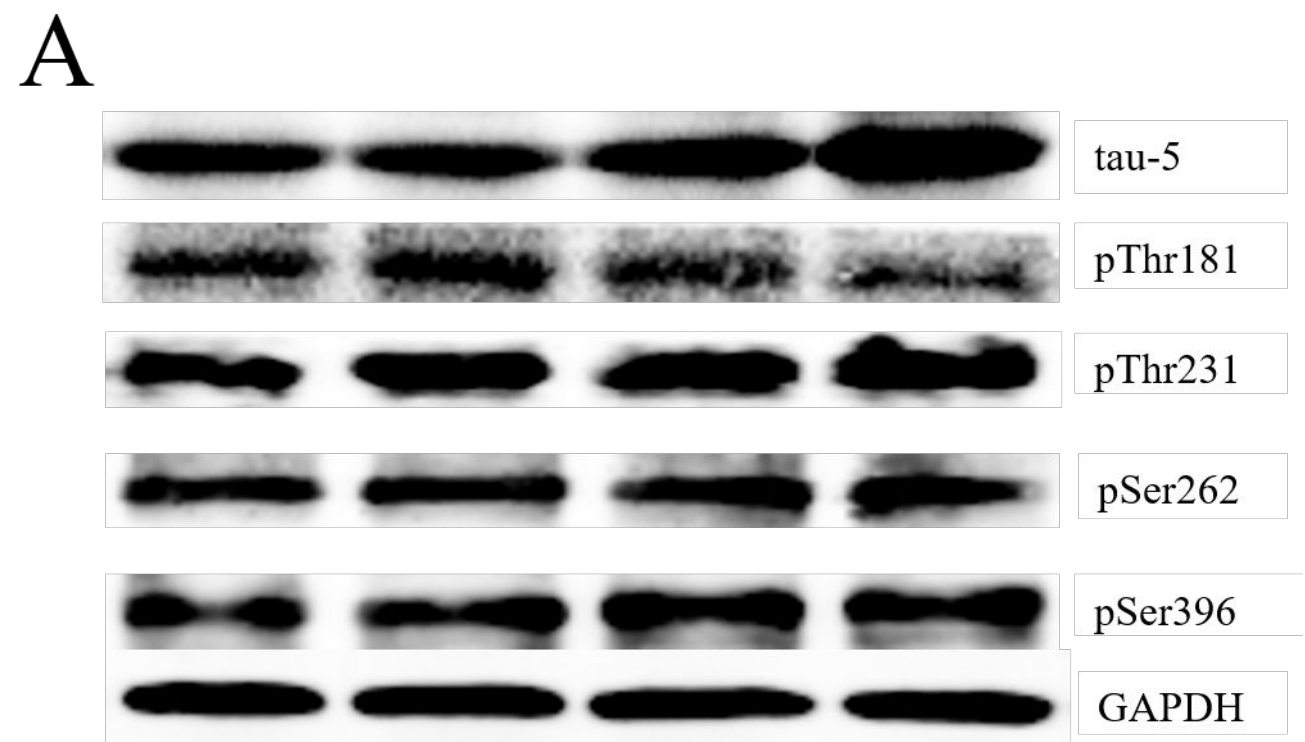

Control $\quad 0.5 \mathrm{mmol} / \mathrm{L} \quad 1 \mathrm{mmol} / \mathrm{L} \quad 2 \mathrm{mmol} / \mathrm{L}$

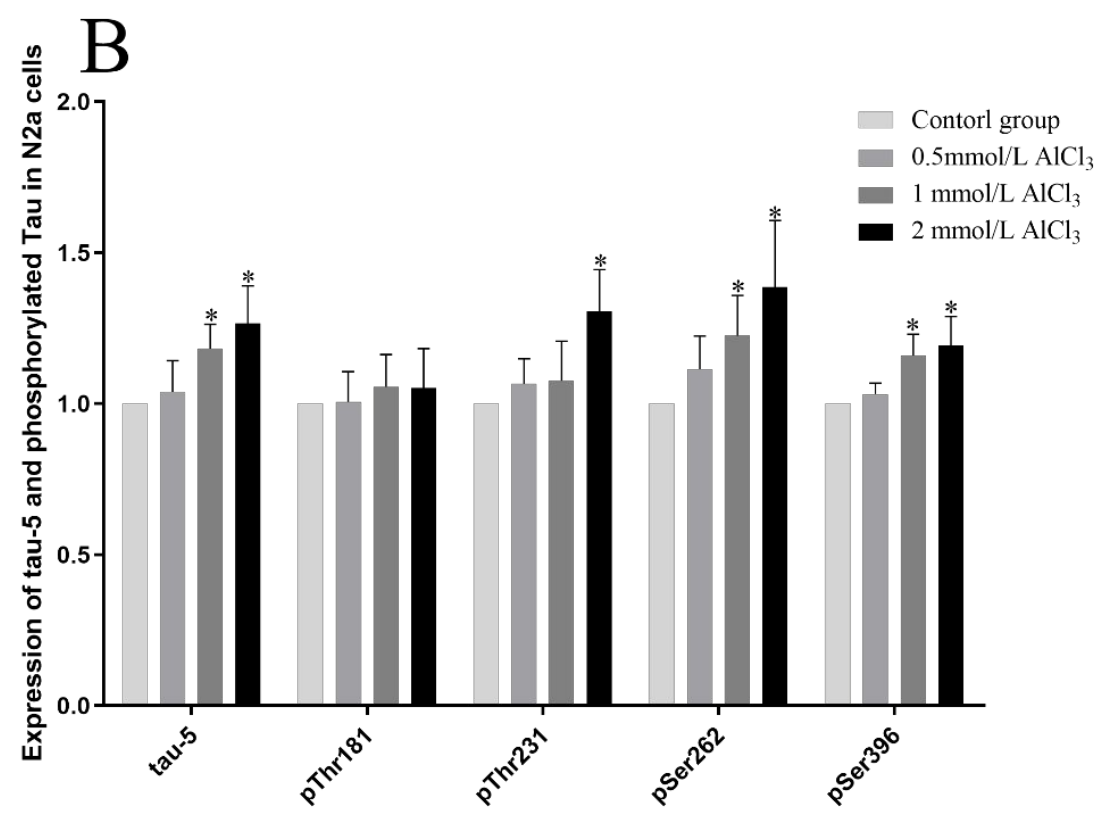

Figure. S1 The expression of tau-5 and phosphorylated Tau protein induced by $\mathrm{AlCl}_{3}$ in N2a cells. With increasing aluminium concentration, the expression of tau-5 increased, and the expression of pThr231, pSer262 and pSer396 increased, but the expression of pThr181 did not change significantly with increasing aluminium concentration (Figure S1 A, B). The statistical analysis results are expressed with 
respect to the reference protein GAPDH after standardization. Compared with the control group, ${ }^{*} P<0.05$.
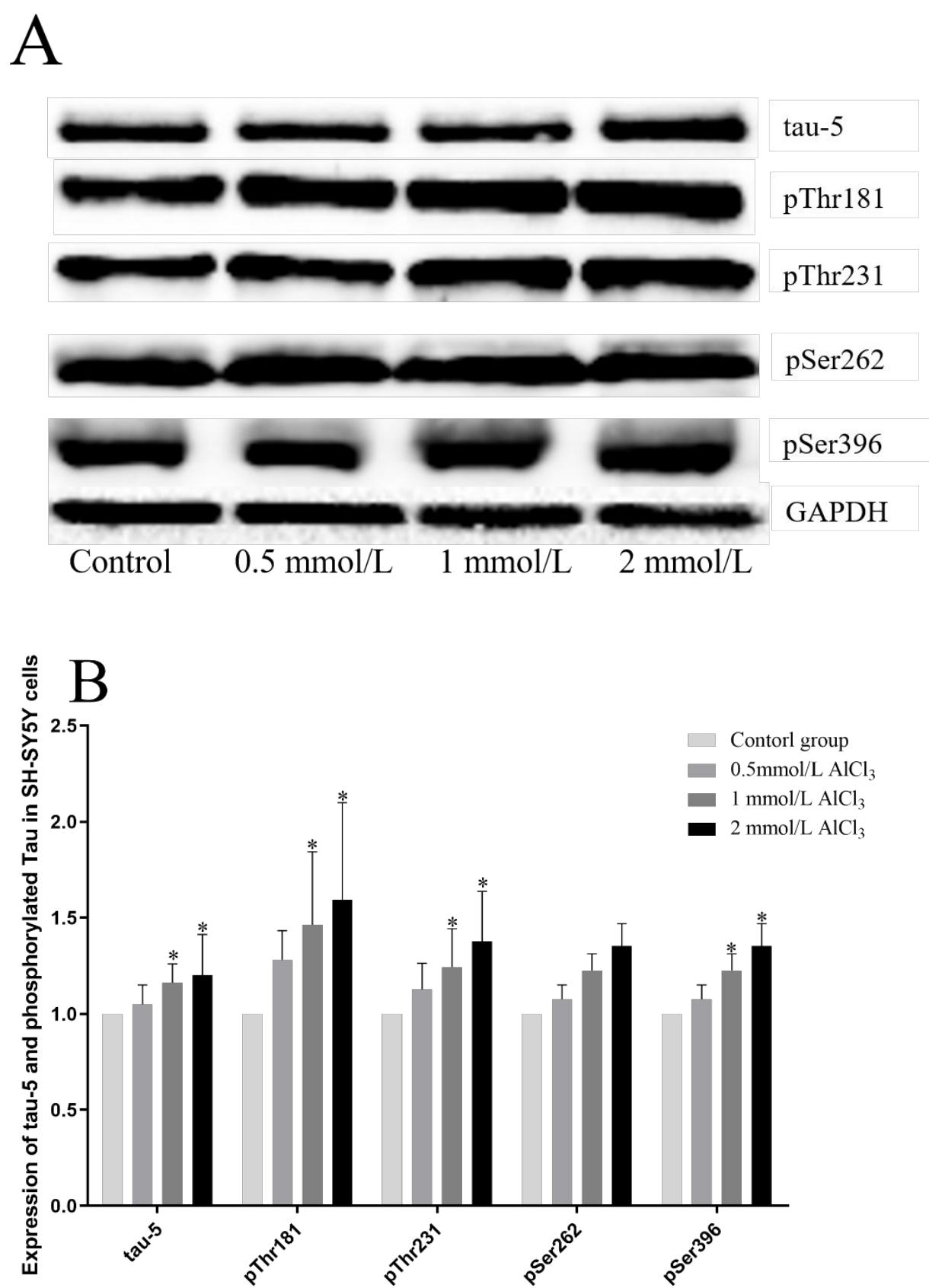

Figure S2 The expression of tau-5 and phosphorylated Tau in SH-SY5Y cells. With increasing aluminium concentration, the expression of tau-5 increased, and the expression of Thr181, Thr231 and Ser396 increased, but the degree of pSer262 did not change significantly with increasing aluminium concentration (Figure S2 A,B). The 
statistical analysis results are expressed with respect to the reference protein GAPDH after standardization. Compared with the control group, ${ }^{*} P<0.05$.
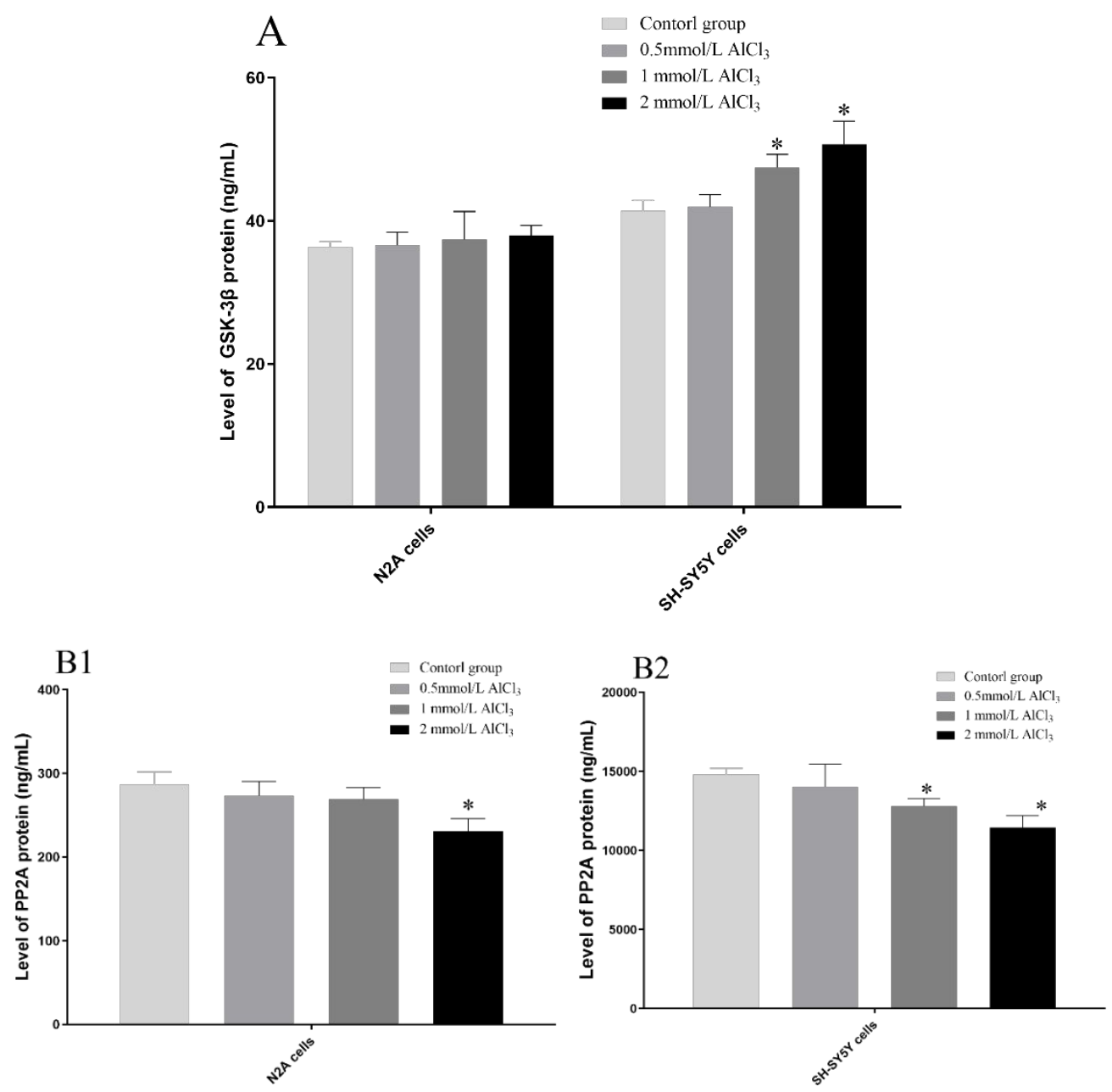

Figure $\mathbf{S 3}$ The level of GSK-3 $\beta$ induced by $\mathrm{AlCl}_{3}$ in $\mathrm{N} 2 \mathrm{a} \& \mathrm{SH}-\mathrm{SY} 5 \mathrm{Y}$ cells (Figure S3 A). The level of PP2A induced by $\mathrm{AlCl}_{3}$ in N2a cells (Figure S3 B1). The level of PP2A induced by $\mathrm{AlCl}_{3}$ in $\mathrm{SH}-\mathrm{SY} 5 \mathrm{Y}$ cells (Figure S3 B2). Compared with the control group, $* P<0.05$. 
A

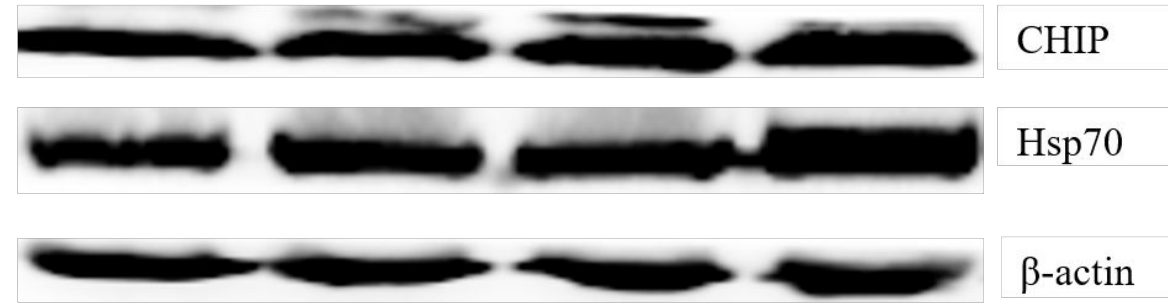

Control $\quad 0.5 \mathrm{mmol} / \mathrm{L} \quad 1 \mathrm{mmol} / \mathrm{L} \quad 2 \mathrm{mmol} / \mathrm{L}$

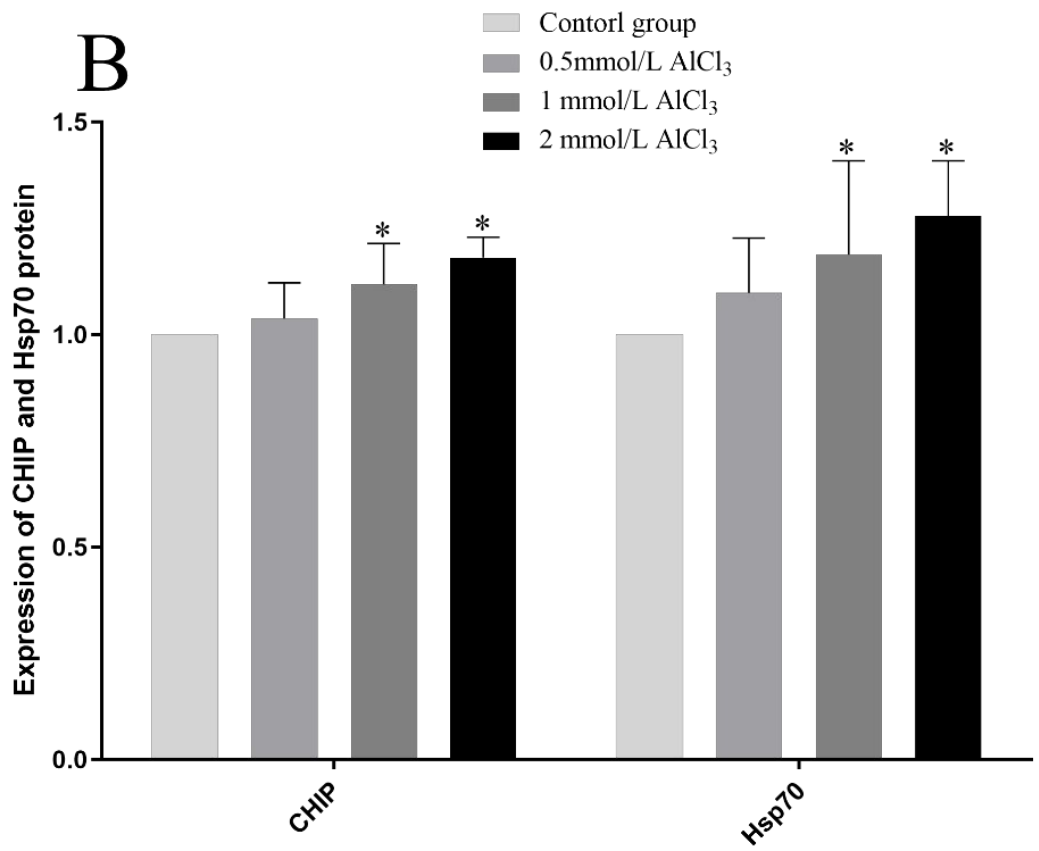




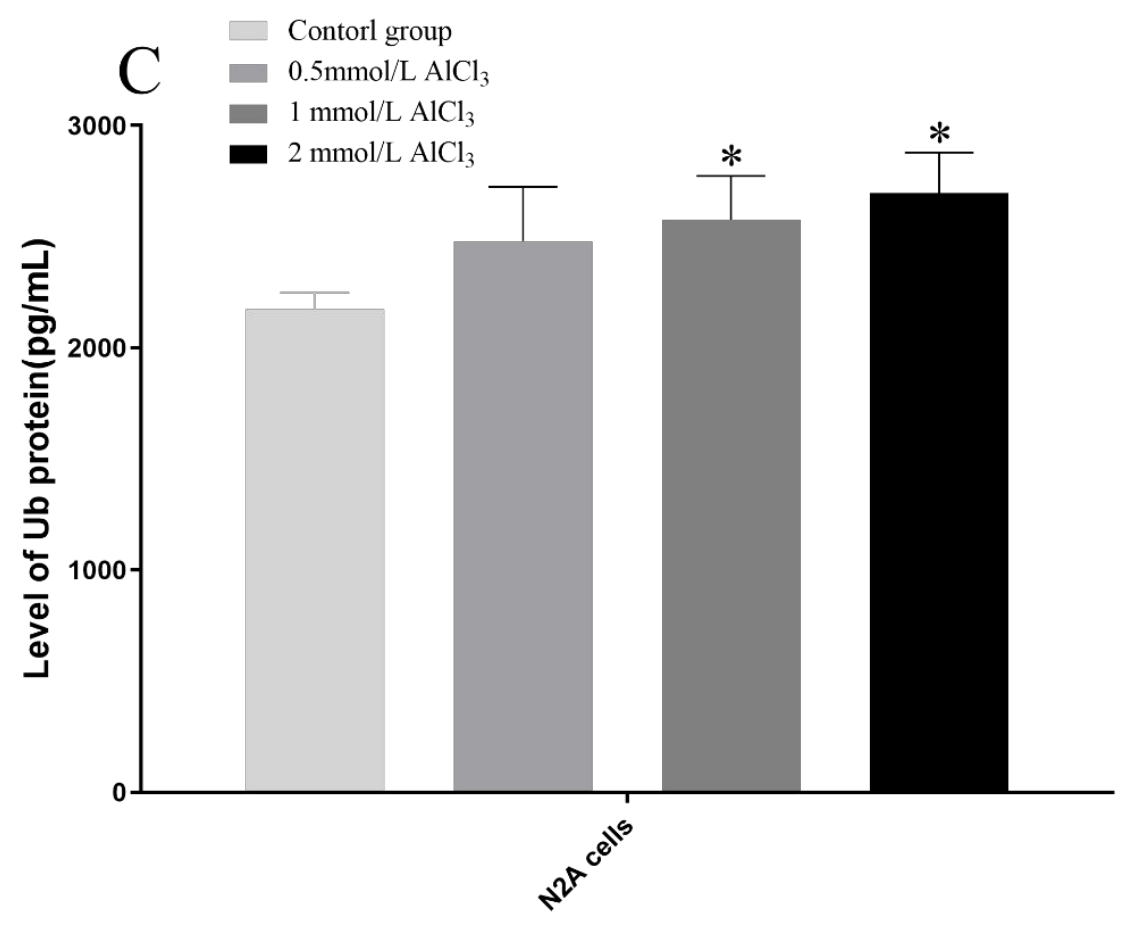

Figure S4 The expressions of CHIP and HSP70 in aluminium-exposed N2A cells (Figure S4 A,B). The level of $\mathrm{Ub}$ in aluminium-exposed N2A cells (Figure S4 C), compared with the control group, ${ }^{*} P<0.05$. 
A

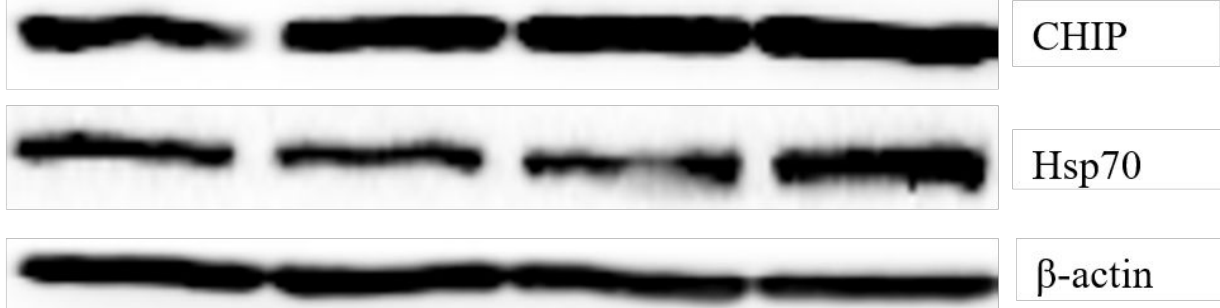

Control $\quad 0.5 \mathrm{mmol} / \mathrm{L} \quad 1 \mathrm{mmol} / \mathrm{L} \quad 2 \mathrm{mmol} / \mathrm{L}$

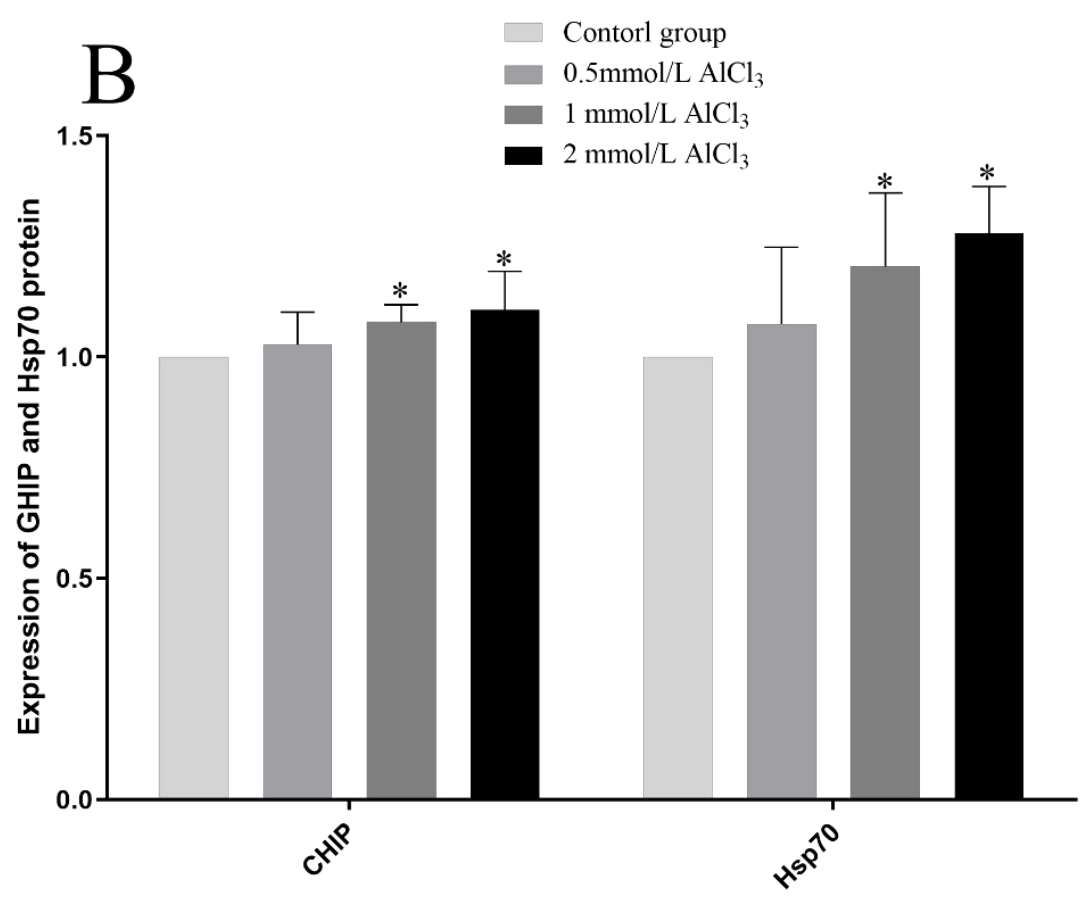




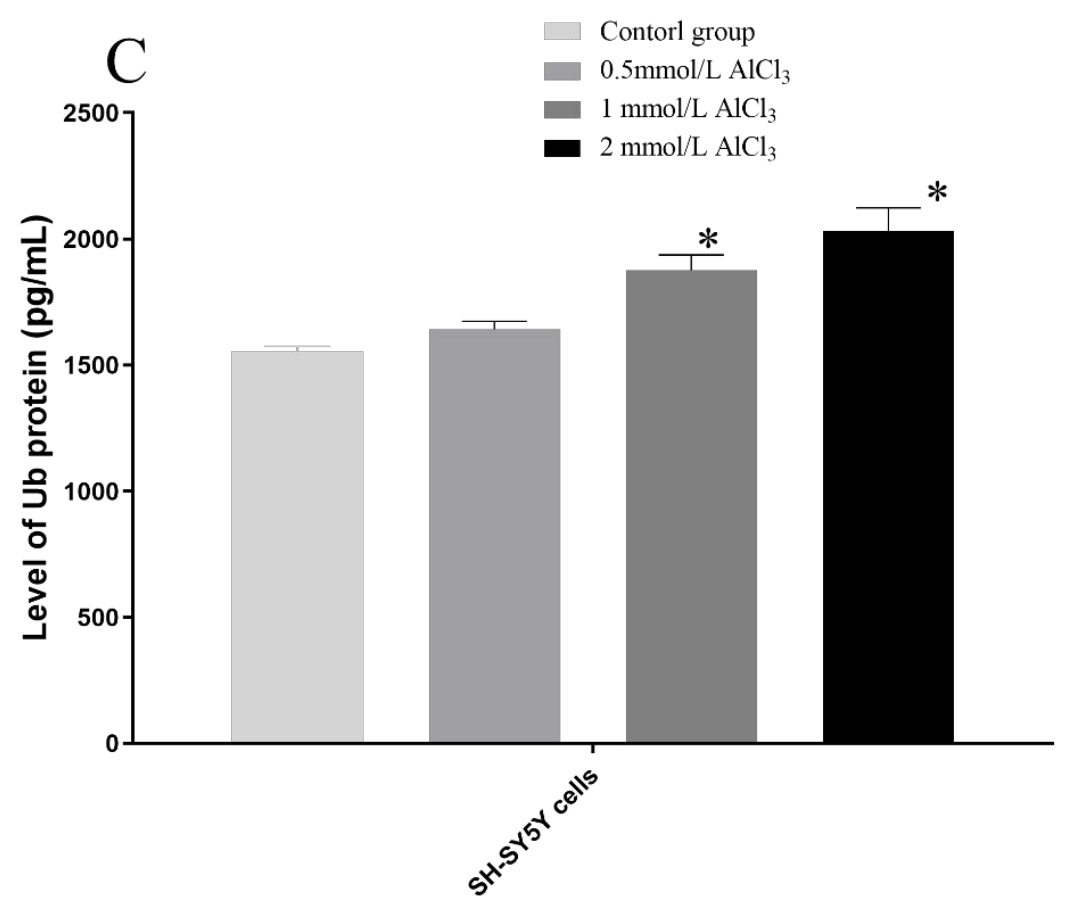

Figure S5 The expressions of CHIP and HSP70 in aluminium-exposed SH-SY5Y cells

(Figure S5 A,B). The levels of Ub in aluminium-exposed SH-SY5Y cells (Figure S5

C). Compared with the control group, ${ }^{*} P<0.05$. 


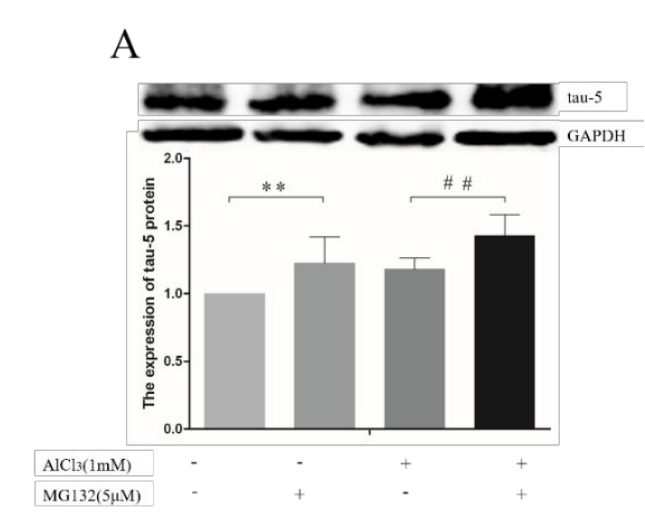

C

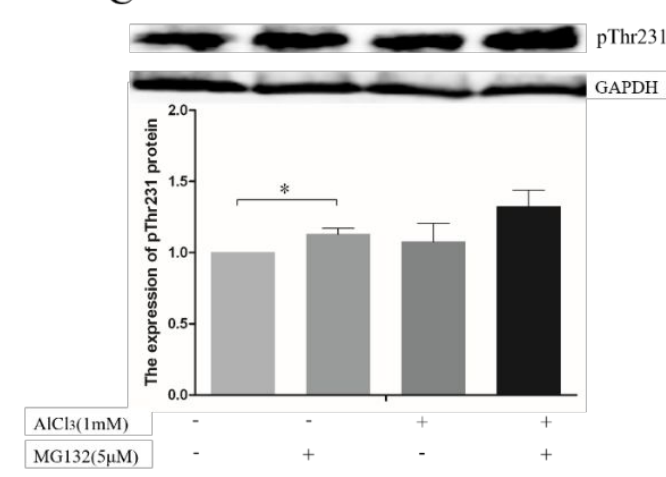

B

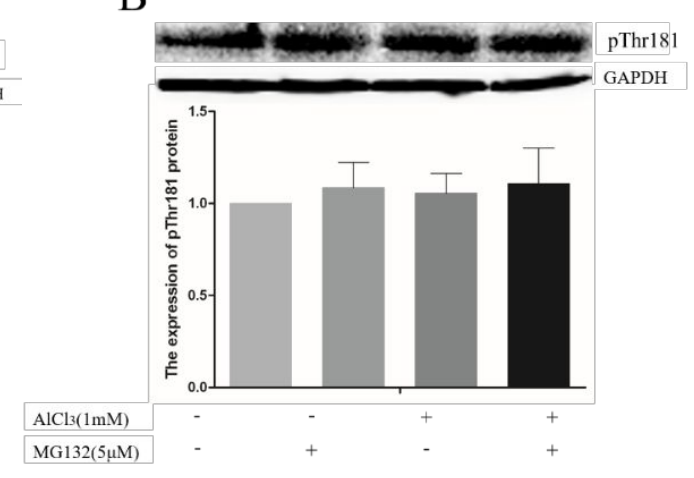

$\mathrm{D}$

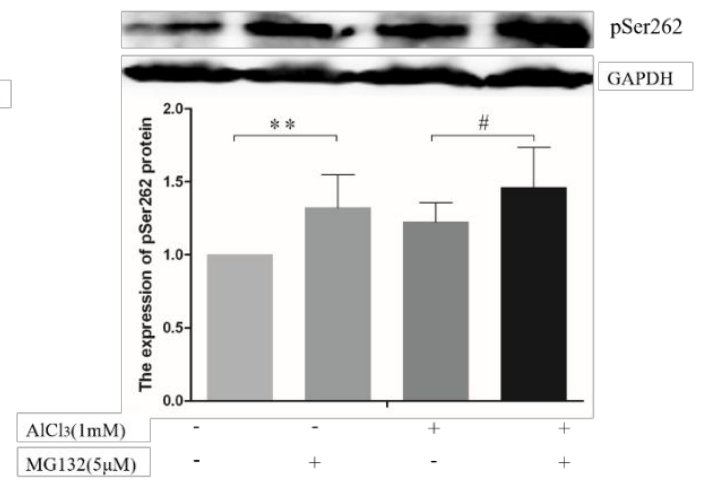

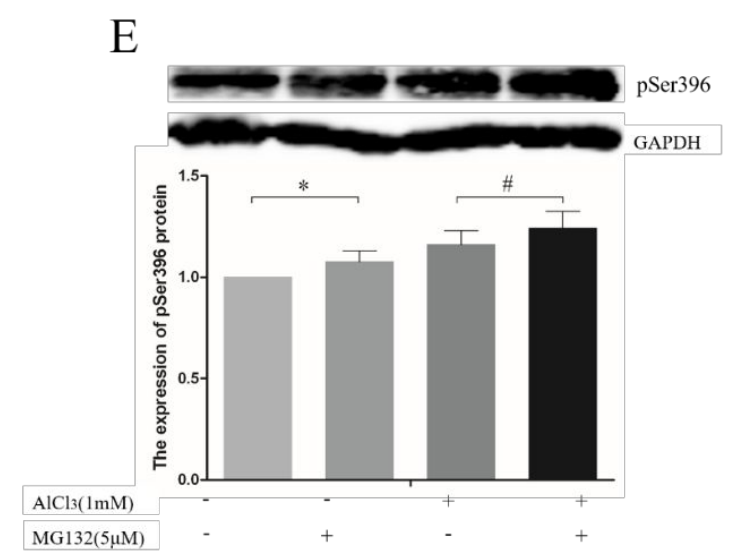

Figure S6 N2a cells were treated with $5 \mu \mathrm{mol} / \mathrm{L}$ MG132 for $6 \mathrm{~h}$ and then treated with $1 \mathrm{mmol} / \mathrm{L} \mathrm{Al}{ }^{3+}$ for $24 \mathrm{~h}$. The expression of tau-5 in N2a cells treated with MG132 (Figure S6 A). The expression of the pThr181 in N2a cells treated with MG132 (Figure S6 B). The expression of the pThr231 in N2a cells treated with MG132 (Figure S6 C). The expression of the pSer262 in N2a cells treated with MG132 (Figure S6 D). The expression of pSer396 in N2a cells treated with MG132 (Figure S6 E). The statistical 
analysis results are expressed with respect to the reference protein GAPDH after standardization. Compared with the control group, ${ }^{*} \mathrm{P}<0.05,{ }^{* *} \mathrm{P}<0.01$; compared with the $1 \mathrm{mmol} / \mathrm{L} \mathrm{Al}{ }^{3+}$ group, ${ }^{\#} \mathrm{P}<0.05,{ }^{\# \#} \mathrm{P}<0.01$.

A

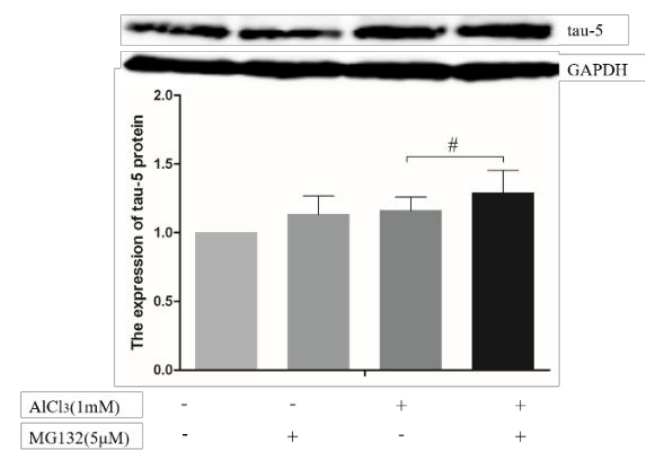

$\mathrm{C}$

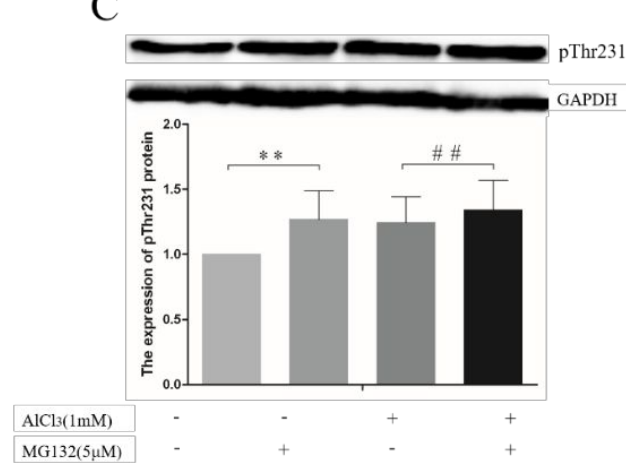

$\mathrm{B}$

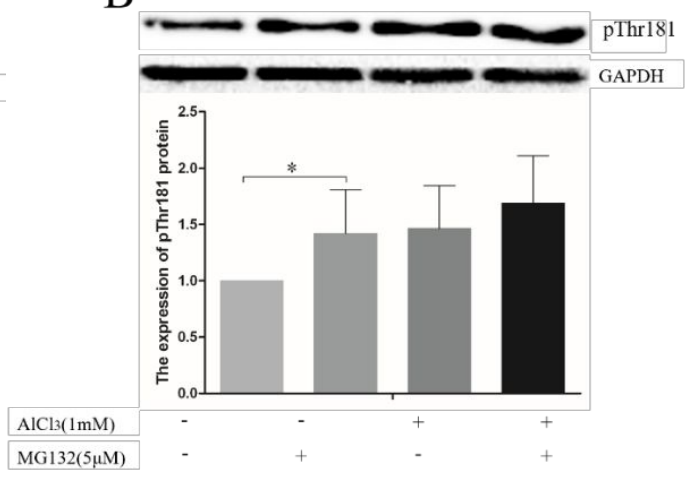

$\mathrm{D}$
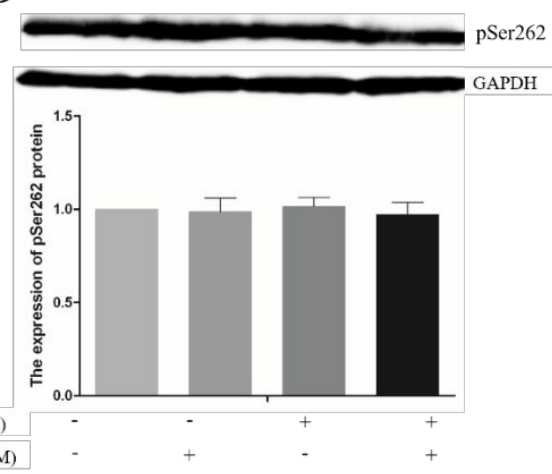

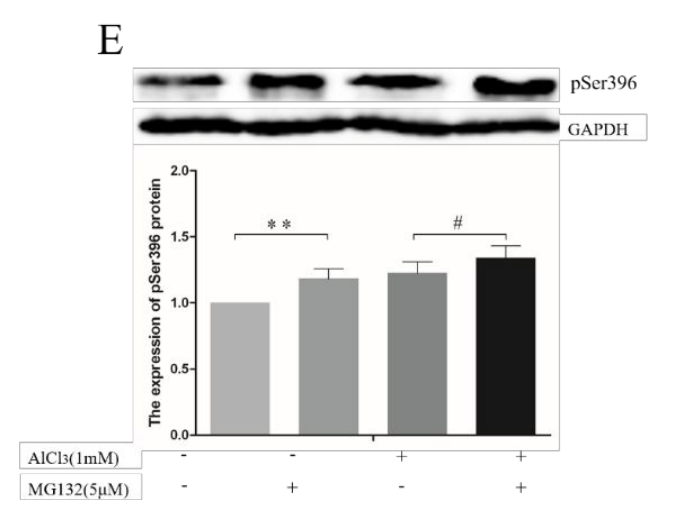

Figure S7 The total tau protein and the phosphorylated tau protein at different sites in SH-SY5Y cells treated with $5 \mu \mathrm{mol} / \mathrm{L} \mathrm{MG132}$ for $6 \mathrm{~h}$ and then treated with $1 \mathrm{mmol} / \mathrm{L}$ $\mathrm{AlCl}_{3}$ for $24 \mathrm{~h}$. The expression of tau-5 in SH-SY5Y cells treated with MG132 (Figure S7 A). The expression of the pThr181 in SH-SY5Y cells treated with MG132 (Figure 
S7 B). The expression of the pThr231 in SH-SY5Y cells treated with MG132 (Figure

S7 C). The expression of the pSer262 in SH-SY5Y cells treated with MG132 (Figure

S7 D). The expression of the pSer396 in SH-SY5Y cells treated with MG132 (Figure

S7 E). The statistical analysis results are expressed with respect to the reference protein

GAPDH after standardization. Compared with the control group, ${ }^{*} \mathrm{P}<0.05,{ }^{* *} \mathrm{P}<0.01$; compared with the $1 \mathrm{mmol} / \mathrm{L} \mathrm{Al}^{3+}$ group, ${ }^{\#} \mathrm{P}<0.05,{ }^{\#} \mathrm{P}<0.01$.

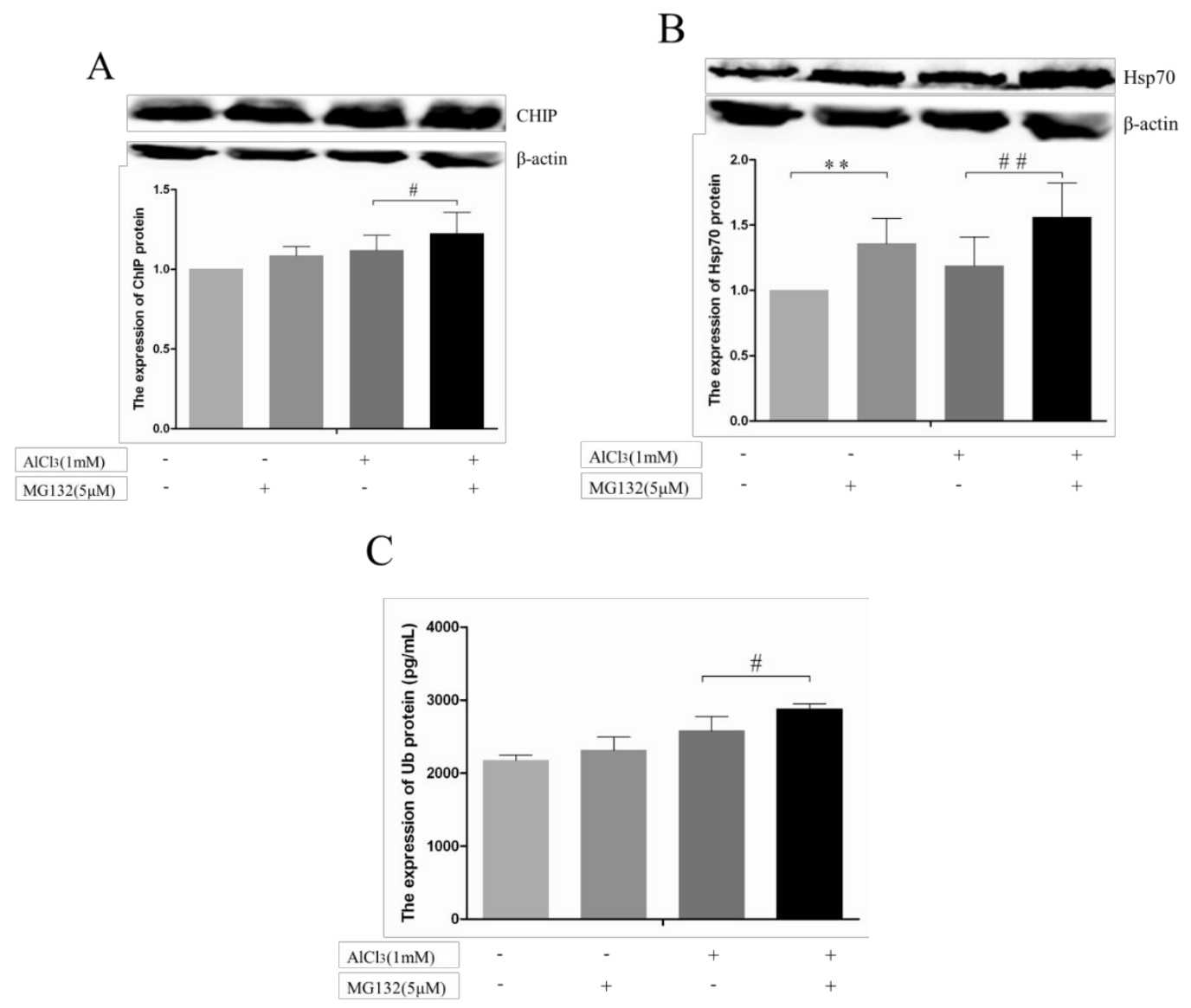

Figure S8 The expression of CHIP and Hsp70, and the level of Ub in N2a cells treated with $5 \mu \mathrm{mol} / \mathrm{L} \mathrm{MG132}$ for $6 \mathrm{~h}$ and $1 \mathrm{mmol} / \mathrm{L} \mathrm{AlCl}_{3}$ for $24 \mathrm{~h}$. The expression of CHIP in N2a cells treated with MG132 (Figure S8 A). The expression of Hsp70 in N2a cells treated with MG132 (Figure S8 B). The level of Ub in N2a cells treated with $5 \mu \mathrm{mol} / \mathrm{L}$ 
MG132 (Figure S8 C). The statistical analysis results are expressed with respect to the reference protein $\beta$-actin after standardization. Compared with the control group, ${ }^{* *} P<0.01$; compared with the $1 \mathrm{mmol} / \mathrm{L} \mathrm{Al}{ }^{3+}$ group, ${ }^{\#} P<0.05,{ }^{\#} P<0.01$.

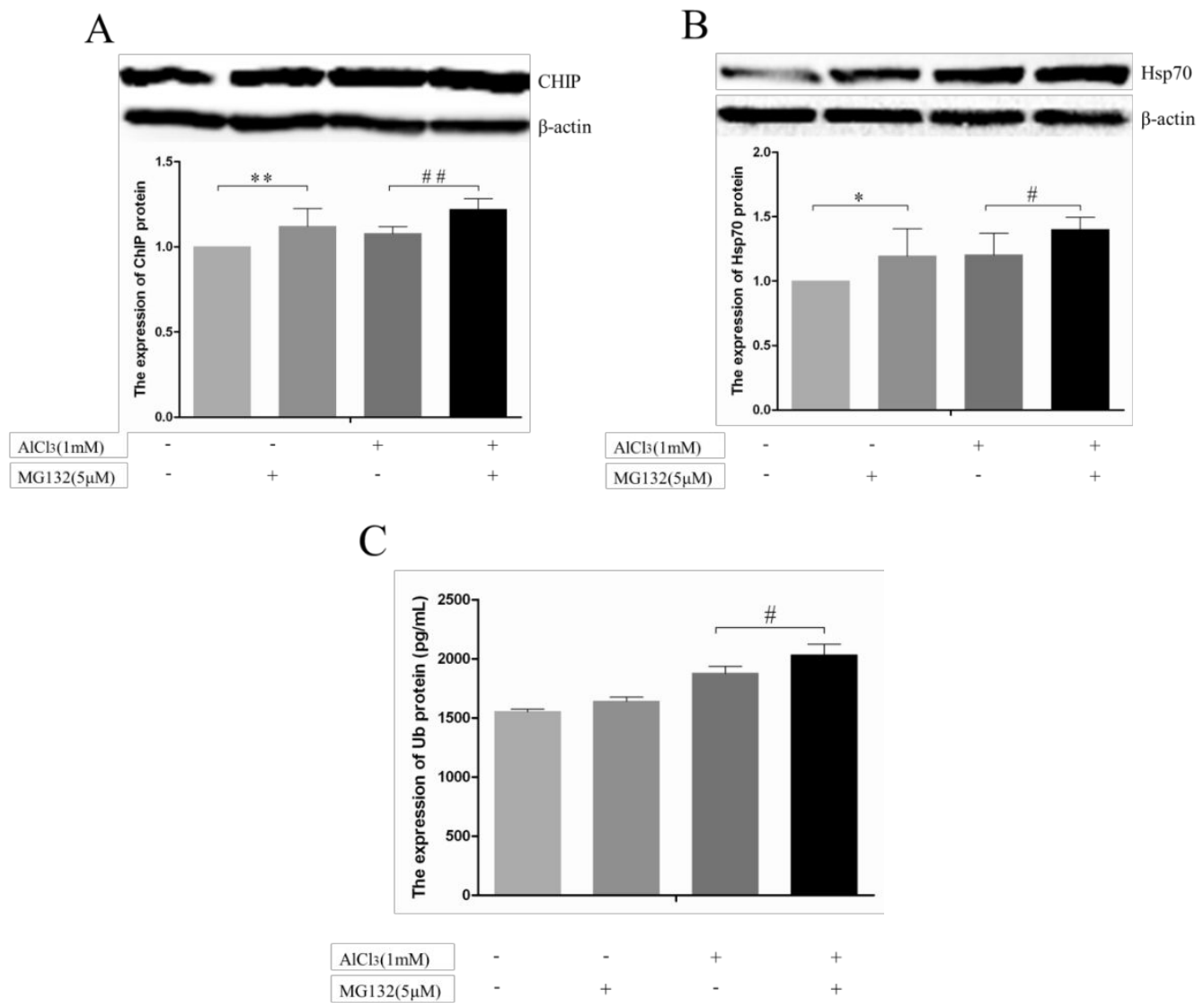

Figure S9 The expression of CHIP in SH-SY5Y cells treated with MG132 (Figure S9 A). The expression of HSP70 in SH-SY5Y cells treated with MG132 (Figure S9 B). The level of Ub in SH-SY5Y cells treated with MG132 (Figure S9 C). Statistical analysis results are expressed with respect to the reference protein $\beta$-actin after standardization. Compared with the control group, ${ }^{*} \mathrm{P}<0.05,{ }^{* *} \mathrm{P}<0.01$; compared with the $1 \mathrm{mmol} / \mathrm{L} \mathrm{Al}{ }^{3+}$ group, ${ }^{\#} P<0.05,{ }^{\#} P<0.01$. 